\title{
Structure formation of black hole jets triggered by electron-scale physics
}

\section{Tomohisa Kawashima ( $\nabla$ kawshm@icrr.u-tokyo.ac.jp)}

Institute for Cosmic Ray Research, The University of Tokyo https://orcid.org/0000-0001-8527-0496

Seiji Ishiguro

National Institute for Fusion Science

\section{Toseo Moritaka}

National Institute for Fusion Science

Ritoku Horiuchi

National Institute for Fusion Science

Kohji Tomisaka

National Astronomical Observatory of Japan

\section{Physical Sciences - Article}

Keywords: black hole, high-energy electrons, relativistic jets

Posted Date: September 3rd, 2020

DOI: https://doi.org/10.21203/rs.3.rs-63856/v1

License: (c) (1) This work is licensed under a Creative Commons Attribution 4.0 International License. Read Full License 


\section{Structure formation of black hole jets triggered by electron-scale physics}

Tomohisa Kawashima $^{1,5}$, Seiji Ishiguro ${ }^{2,4}$, Toseo Moritaka ${ }^{2,4}$, Ritoku Horiuchi $^{2}, \&$ Kohji Tomisaka ${ }^{1,3}$

${ }^{1}$ Center for Computational Astrophysics, National Astronomical Observatory of Japan, National Institutes of Natural Sciences, 2-21-1 Osawa, Mitaka, Tokyo 181-8588, Japan

${ }^{2}$ Fundamental Physics Simulation Research Division, National Institute for Fusion Science, National Institutes of Natural Sciences, 322-6 Oroshi-cho, Toki, 509-5292, Japan.

${ }^{3}$ Division of Science, National Astronomical Observatory of Japan, National Institutes of Natural Sciences, 2-21-1 Osawa, Mitaka, Tokyo 181-8588, Japan

${ }^{4}$ Department of Fusion Science, SOKENDAI (The Graduate University for Advanced Studies), 322-6 Oroshi-cho, Toki 509-5292, Japan

${ }^{5}$ Institute for Cosmic Ray Research, The University of Tokyo, 5-1-5 Kashiwanoha, Kashiwa, Chiba 277-8582, Japan

Jets are ubiquitous in the universe $\mathrm{e}^{1,2}$. The radio jet is a beamed plasma flow with relativistic speed accelerated by a supermassive black hole in some galaxies. Recent observations of the relativistic jet in the elliptical galaxy M87 have discovered a triple-ridge sub-structure in the jet at a distance of one thousand Schwartzchild radius from the black hole ${ }^{3,4}$. The triple-ridge structure may be the first strong evidence of the spine-sheath structure consisting of the fast spine region (jet spine) and relatively slow sheath region (jet sheath), which was hypothetically introduced to explain observational features in various unresolved relativistic jets ${ }^{5,6}$. 
However, the formation mechanism of such a spine-brightened jet is quite enigmatic. Here we report that the combination of the magnetic pinching force induced by an electron-scale shear-instability called Mushroom instability ${ }^{7}$ and the high-energy electron ejections by the subsequent magnetic reconnection leads to the drastic accumulation of high-energy electrons in the center of the cylindrical jet. The concentration of high-energy electrons towards the jet center indicates the appearance of bright jet-spine as observed in M87. Thus, the electronscale, microscopic processes would play an important role in the structure formation and generation of high-energy components in the relativistic jets.

The elliptic galaxy M87 is one of the best laboratories to study the relativistic jet because it possesses one of the closest jets, so that its jet structure is best resolved ${ }^{8,9}$. Recently, very long baseline interferometer (VLBI) observation at $15 \mathrm{GHz}$ with Very Long Baseline Array (VLBA) revealed the triple-ridge structure in M87 jet at a distance of $r \gtrsim 10^{3} r_{\mathrm{S}}$ from the central supermassive black hole ${ }^{3,4}$. Here, $r_{\mathrm{S}}=2 G M / c^{2}$ is the Schwarzschild radius of the central black hole with its mass $M, G$ is the gravitational constant, $M_{\odot}\left(=1.99 \times 10^{33} \mathrm{~g}\right)$ is the mass of the sun, and $c$ is the speed of light. Recent observations of black-hole shadow in M87 with Event Horizon Telescope determined the mass of the black hole to be $M \sim 6.5 \times 10^{9} M_{\odot}{ }^{10}$, hence $r_{\mathrm{S}} \sim 2 \times 10^{15} \mathrm{~cm}$. The triple-ridge structure can be interpreted as the observational feature of the spine-sheath structure of the jet, which is the combination of the fast spine and the slow sheath parts of the jet ${ }^{5}$. The spine-sheath structure is also proposed to explain the short-time variation of $\mathrm{TeV} \gamma$-ray emission in relativistic jets propagating almost parallel to the line-of-sight of observers called blazar (e.g., Mrk 501 and PKS 2155-304) and may be ubiquitous in the relativistic jet ${ }^{6}$. 
In order to understand the physics and the observed features of the relativistic jets, a number of magnetohydrodynamic (MHD) simulations have been performed up to today ${ }^{11-20}$. The general relativistic MHD simulations may reproduce the sheath structure ${ }^{18}$, but could not explain the formation of the jet spine. The observations of jets in supermassive black holes also indicate that the electron energy-spectra in the jet spine are harder than those in the jet sheath ${ }^{6}$. However, the formation mechanism of the jet spine, which is composed of high-energy electrons, is still an important question.

Electron-scale, microscopic processes may play an important role on the structure formation and dynamics in the relativistic jets. Meanwhile, a limited number of particle-in-cell (PIC) simulations of jets have been carried out as a strong tool to explore the electron-scale microscopic processes of jets $^{21-23}$. In the 2010s, an important electron-scale shear-instability called Mushroom instability $(\mathrm{MI})^{24}$ was found in the plasma with relativistic bulk shear flow. Importantly, the MI dominates the electron-scale Kelvin-Helmholtz instability when relative bulk speed is greater than $\sim 0.3 c^{7}$. Based on kinetic plasma simulations ${ }^{7,24-27}$, the mechanism of MI is the following: At first, the interchanging thermal motion of electrons across the shear surface, which is faster than proton motions, results in the electric currents along the jet bulk-motion near the shear surface. Then, the magnetic field is generated mainly parallel to the shear surface. The generated magnetic field further induces the interchanging electron motion across the velocity shear surface.

We propose that, in jets with cylindrical velocity-shear surface, the interchange motion across the shear surface tends to the electron concentration to the center (i.e., spine) of the jet via the 
cylindrical geometric effects. In addition to the concentration of the electrons, the simultaneous acceleration of the electron, which is indicated by the observation of relativistic jets, should be also explained. Here, we perform fully kinetic PIC simulations of the relativistic jets of fully ionized hydrogen plasma to explore the mechanism which can simultaneously explain the formation of the spine-structured jet and the acceleration of the electrons there. The simulations are carried out by using a two-dimensional PIC simulation code PASTEL ${ }^{28}$ in the Cartesian coordinate system with $x-y$ plane, which can explicitly solve the Maxwell equations and the Newton-Lorentz equation in a self-consistent way.

The initial condition of the simulation is as follows. The cylindrical jet with bulk speed $v_{\text {bulk }}=0.9 c$ in the positive $z$ direction is located at the center of the computational domain $(x$ $y$ plane). The diameter of the jet is set to be $100 \mathrm{c} / \omega_{\text {pe }}$, where $\omega_{\text {pe }}$ is the plasma frequency of electrons. The temperatures of the electrons and protons are constant in space, and their thermal velocities equal to $0.1 c$ and $0.025 c$, respectively. The computational particles called "superparticles" are also uniformly distributed and neither magnetic field nor electric current is assumed.

In Fig. 1, we show the synthetic picture of our jet model resulting from the PIC simulation. The MI generates and amplifies the magnetic field, while the magnetic field is initially zero. The electrons concentrate in the center of the jet region, as the relativistic jet propagates. This is due to the combination of magnetic pinching force induced by MI and the high energy electron ejections by the subsequent, episodic magnetic reconnection (MR), where MR is the topological rearrangement of magnetic fields with dissipation of magnetic energy and resultant heating and 
acceleration of plasma particles ${ }^{29-35}$. Hereafter, we refer to this new type MR as MI-driven-MR. As a consequence, the number density multiplied by the magnetic field strength, which roughly reflect the synchrotron emissivity, becomes higher as the jet propagates (i.e., as the simulation time increases). As is shown in later, MI-driven-MR accelerates the electron especially inside the initial velocity-shear surface. This confirms that the region inside the velocity shear surface becomes bright via synchrotron emission as the jet propagates, which proposes the formation mechanism of the jet spine.

Next, we present the detail of MI-driven-MR near the velocity-shear surface. Figure 2 displays magnified view of the electron density, transverse electron velocity, electric current density, and energy conversion rate to electrons at $t=2.5 \times 10^{2} \omega_{\mathrm{pe}}^{-1}$. As a result of the nonlinear evolution of the MI, an elongated current sheet surrounded by anti-parallel magnetic fields grows in the radial direction. The direction of the electric current density is anti-parallel to the bulk-jet motion, since the electric current is generated via the MI. Importantly, X-shaped singular points of magnetic field lines ("reconnection points") appear at the center of the current sheet, in which the reconnection component (z-component) of electric field is generated along the electric current density through a microscopic kinetic process ${ }^{36}$, and thus the field energy is strongly converted to electrons. In other words, the subsequent heating and acceleration of the electron in the transverse direction of the jet occur inside the current sheet via the MI-driven-MR. In addition, the magnetic field in the $z$-direction also appears and the acceleration in the $z$-direction becomes important, especially in the late phase of the simulation as a consequence of the coupling with the effect of the generation of the return current in the jet spine. Another strong evidence of MR is shown by the appearance 
of the hole structure in the phase-space of electrons as shown in supplementary information.

The high-energy electrons concentrate to the jet center as the episodic MI-driven-MRs evolve. In Figure 3, we show time evolution of the MI-driven-MRs. One can find that the reconnection point moves towards the jet center as time increases, which results in the contraction of the current sheet surrounding the jet center. This is because the electrons which generate the electric current move towards the jet center due to the MRs and the Lorentz force as a consequence of MI. As the current sheet contracts, the reconnection point moves towards the jet center. Then, the accelerated and/or heated electrons concentrate in the jet center, i.e., the jet spine is formed.

The MI-driven-MR efficiently accelerates electrons. Figure 4 displays the time evolution of electron energy-spectra in the whole computational domain. Initially, the electrons outside the velocity-shear surface shows the Maxwell distribution, while the shifted-Maxwellian appears inside the shear surface because the electrons have the bulk velocity with $v=0.9 c$. One can find that the more electrons are accelerated with time via MI-driven-MR and the power-law spectra with narrow energy range appear as explained below.

Just after the MI grows, the electrons outside the shear surface are mainly accelerated and the Maxwell distribution starts to be distorted around $\gamma-1 \sim 0.1$ at $t \gtrsim 20 \omega_{\mathrm{pe}}^{-1}$. The MRs intermittently take place with their reconnection points, which move towards the center of the cylindrical jet with time as shown in Figure 3. At $t=750 \omega_{\mathrm{pe}}^{-1}$, the power-law spectra with narrow energy range is formed at $\gamma-1 \simeq 1$. As shown in the right panel of Figure 4 , the highly accelerated particles are inside the initial shear surface, indicating that the jet spine is composed of high energy 
electrons. At the final stage of our simulation, the electrons are accelerated in the jet direction $(z-$ direction), while the particles are accelerated to the transverse direction ( $x$ - and/or $y$-direction) in the early non-linear stage of the simulation.

In terms of the timescale, the concentration of electrons in the jet triggered by MI is plausible. The typical propagation timescale of the relativistic jet up to the distance with appearance of tripleridge structure for M87 $\left(\sim 10^{3} r_{\mathrm{S}}\right)$ is $t_{\mathrm{dyn}} \gtrsim 10^{3} r_{\mathrm{S}} / c \sim 10^{8} \mathrm{~s}$. Next, the growth timescale of MI $t_{\mathrm{MI}}$ can be estimated below. The growth timescale of MI is $t_{\mathrm{MI}} \sim 1 / \omega_{\mathrm{pe}}$ (see the supplementary information in detail). The plasma frequency of electrons $\omega_{\mathrm{pe}}=\sqrt{4 \pi e^{2} n_{\mathrm{e}} / m_{\mathrm{e}}}$ can be estimated to be $\sim 10^{6} \sqrt{n_{\mathrm{e}} / 10^{4} \mathrm{~cm}^{-3}} \mathrm{~s}^{-1}$, where we can roughly speculate the electron number density of the jet is $n_{\mathrm{e}} \lesssim 10^{4} \mathrm{~cm}^{-3}$ since the synchrotron flux from the jet should be less than the observed flux in M87 by EHT observations ${ }^{37}$. Therefore, $t_{\mathrm{MI}} \gtrsim 10^{-6} \mathrm{~s}$ and this is greatly less than the propagation timescale of the relativistic jet $t_{\mathrm{dyn}}$. In practice, the formation timescale of jet spine is longer than the MI growth timescale. In our simulation, the electron density near the jet center significantly increases after the timescale at which the electrons with thermal velocity $(0.1 c)$ reaches the jet center from the velocity shear surface $\left(50 c / \omega_{\text {pe }}\right)$, i.e., $\sim 500 / \omega_{\text {pe }}$ Since the radius of M87 jet at $\sim 10^{3} r_{\mathrm{S}}$ from the $\mathrm{BH}$ is $\sim 50 r_{\mathrm{S}} 4,38,39$ and the thermal velocity of electrons are expected to be $\gtrsim 0.1 c$, the formation timescale of the jet spine is estimated to be $\lesssim 10^{7}$ s. Hence, with respect to the timescale, our scenario of the jet-spine formation via MI-driven-MR is consistent with the observed M87 jet.

The results of our PIC simulations indicate that the number density of the electrons can be 
high in the center of the jet. In other words, the bright jet spine, which was found in the recent VLBI observations, can be formed. In addition, the electrons are highly accelerated in the spine region. Evidence of the MI-driven-MRs would be found in the future VLBI observation of the polarized radio images at $10^{3} r_{\mathrm{S}}$. If the MI-driven-MRs take place in the relativistic jets, we expect that the azimuthal component of magnetic fields surrounding the jet spine will be generated via the MIs. Since the MHD simulations, i.e., the plasma dynamics without MI-driven-MRs, suggest that the magnetic field near the jet axis would be almost along the direction of the jet propagation, future detection of the strong azimuthal component of magnetic fields in the jet spine will be a smoking gun of our new scenario of jet-spine formation.

\section{Methods}

We perform fully kinetic PIC simulations ${ }^{40-42}$ of the relativistic jets of electron-proton plasma to probe the formation mechanism of the jet spine and the acceleration of the electrons simulta-

neously. The simulations are carried out by using a 2D3V (two dimensional in real space and three dimensional in velocity space) PIC simulation code PASTEL ${ }^{28}$ which explicitly solves the Maxwell equations and the Newton-Lorentz equation.

We solve the kinetic equations of electron-proton plasma in the transverse-direction to the jet propagation in order to study the formation of the jet spine and the acceleration mechanism of the electrons. We set the mass ratio of protons to electrons to be 1836 , i.e., the real mass ratio. In our 2D3V PIC simulations, the Cartesian coordinate is employed: the simulation domain is on the $x-y$ plane and we set the $z$ axis to be the direction of the jet propagation. The size of the 


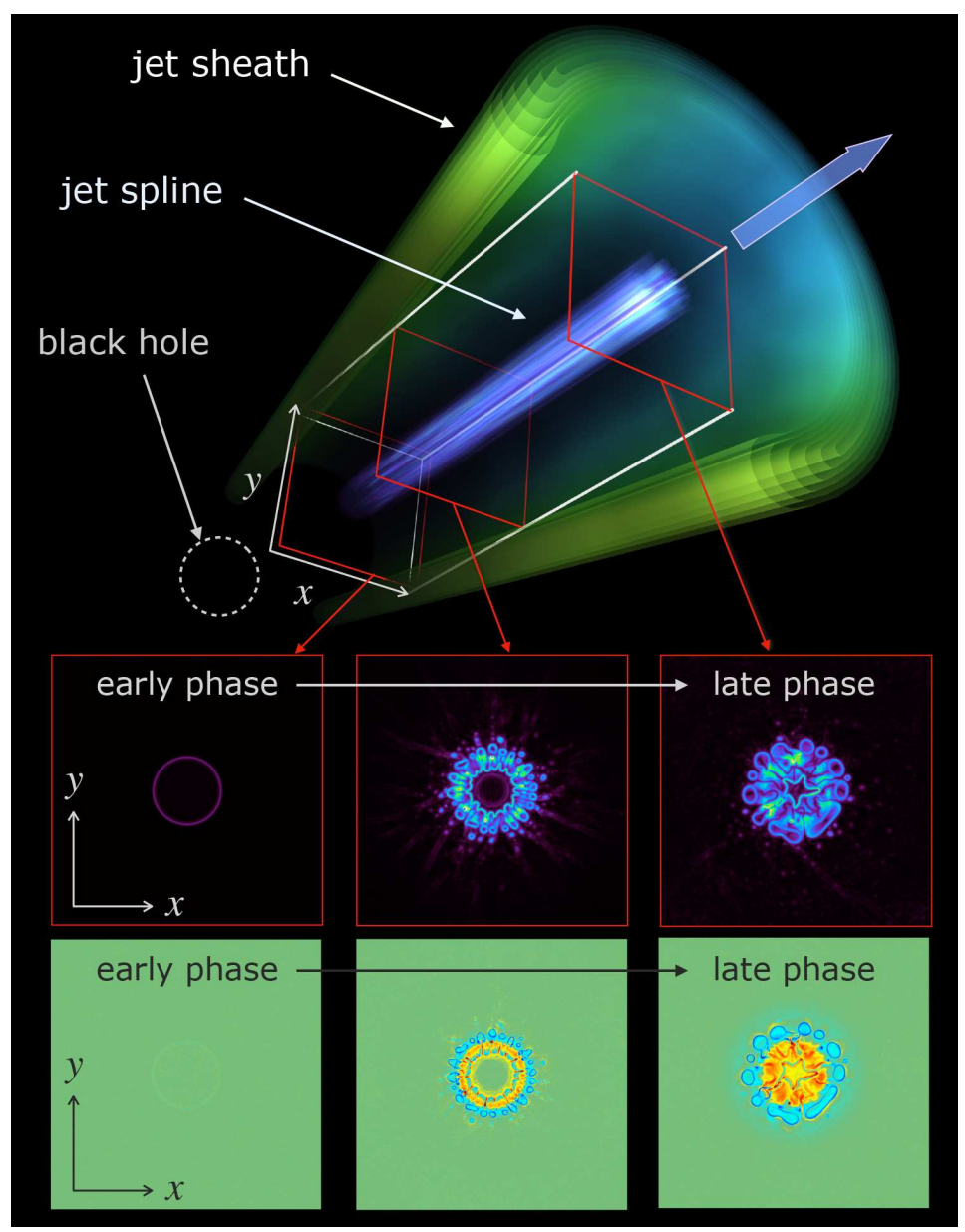

Figure 1: Synthetic picture of our relativistic jet model. The top panel is composed of the volume rendered image of our PIC simulation (jet spine, which is colored in blue-green-white) and schematic pictures (jet sheath colored in green). This image is synthesized by stratifying the time snapshots of our 2D PIC simulation in the direction of the jet propagation. The color represents the electron density multiplied by the strength of the magnetic field, which roughly agrees with the synchrotron emissivity, which is the most important radiative process in the M87 jet. The middle and bottom panels show the spatial distribution of the electron-density multiplied by the strength of the magnetic field the above pseudo synchrotron emissivity and the electron-density itself only, respectively, at each epoch. (animation: supplementary material) 

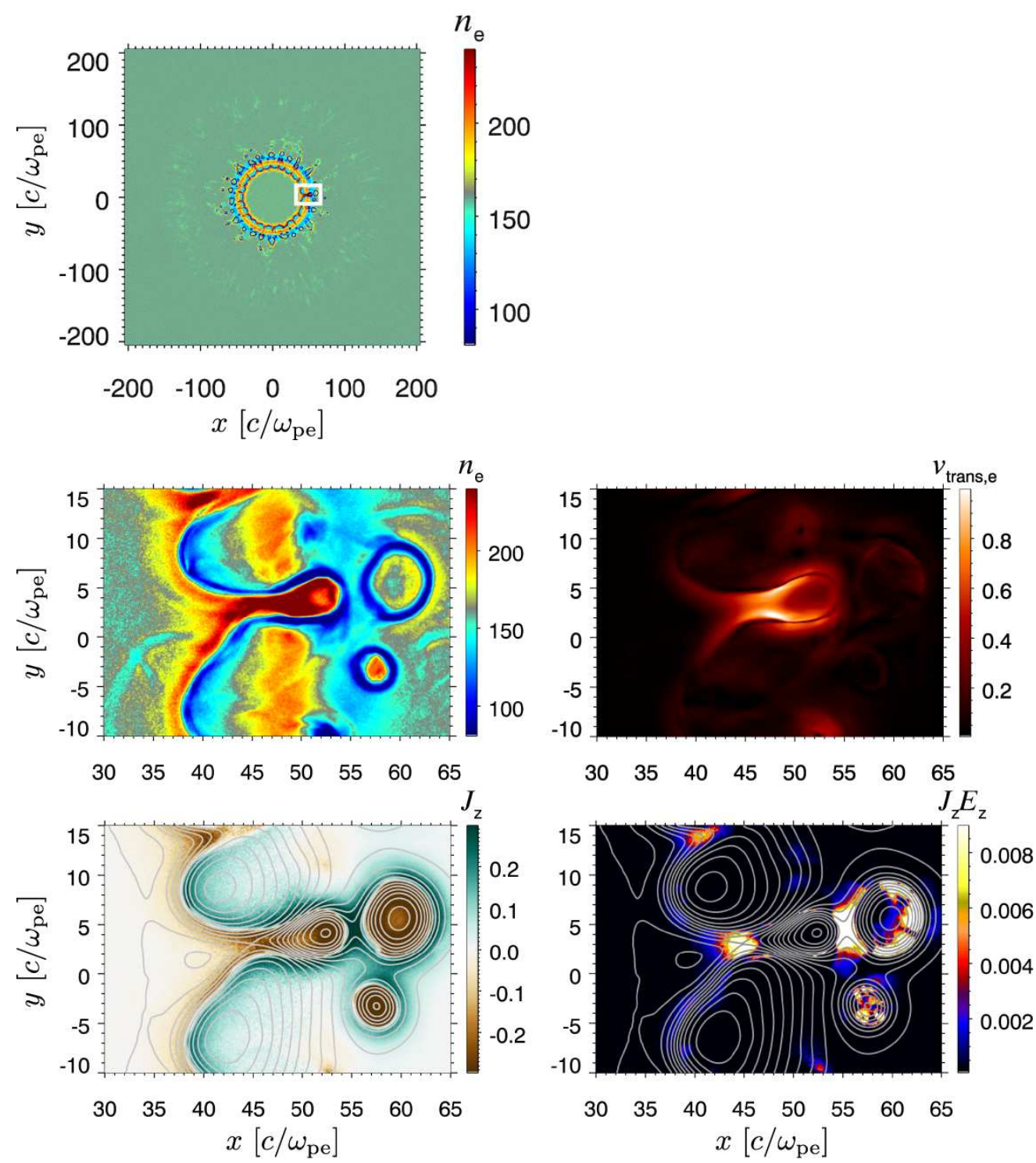

Figure 2: Electron density map in the whole simulation domain (top) at $t=2.5 \times 10^{2} \omega_{\text {pe }}^{-1}$. Magnified view of the electron density (middle left), the absolute value of the four-vector of the electron velocity in the transverse direction of the jet (middle right), $z$ component of electric current density (bottom left), and the product of $z$ component of electric current density and electric field (bottom right) in the region enclosed in the top panel. The electric current and the electric field are evaluated in the co-moving frame of the electrons at each point. The gray lines in the bottom panels display the magnetic field lines in the plane. (animation: supplementary material) 


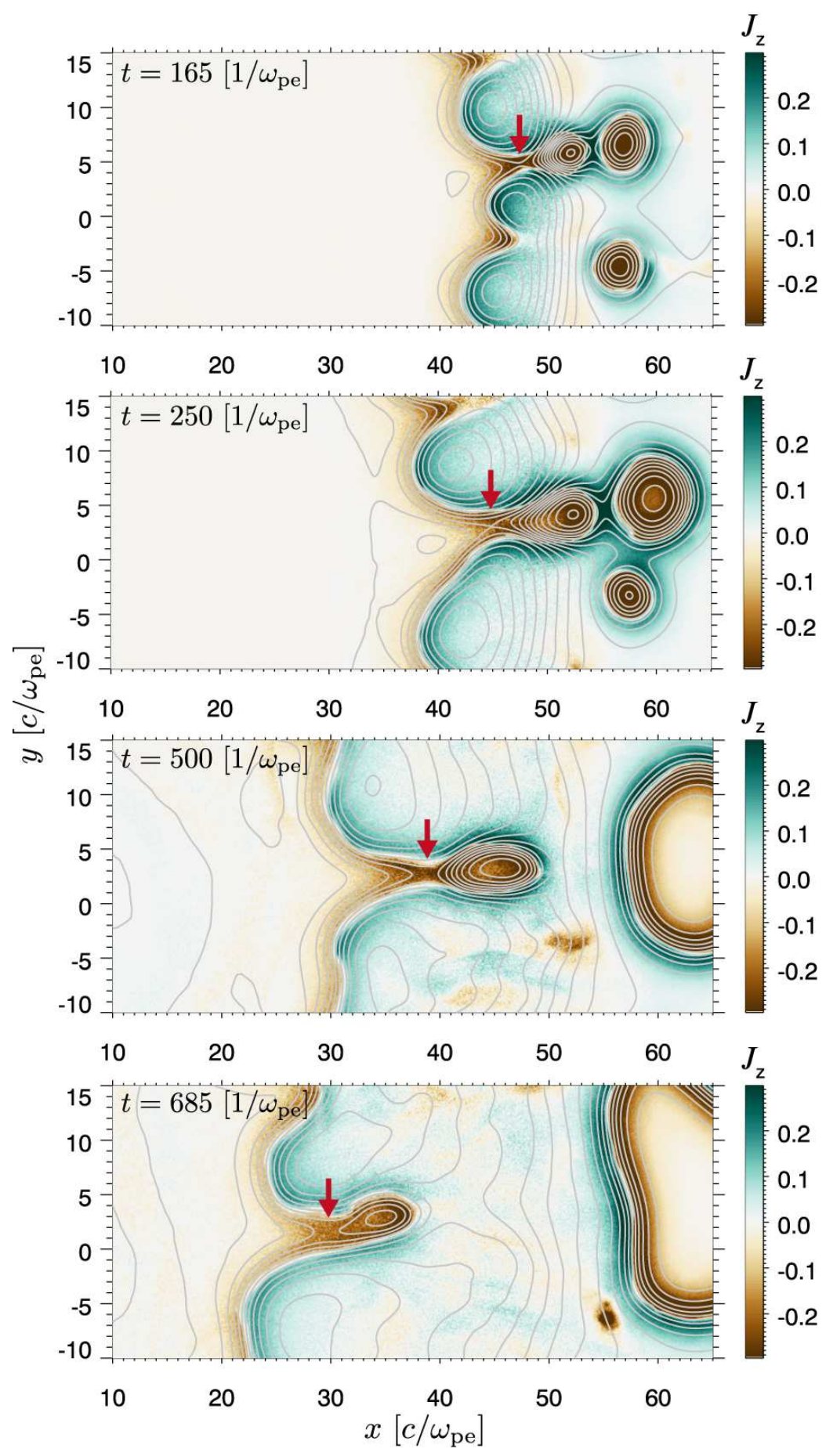

Figure 3: Time evolution of the MI-driven-MR near shear surface. The electric current sheet and the reconnection points (red arrows) shift towards the jet center with time. The electric current is evaluated in the co-moving frame of electrons at each point. 

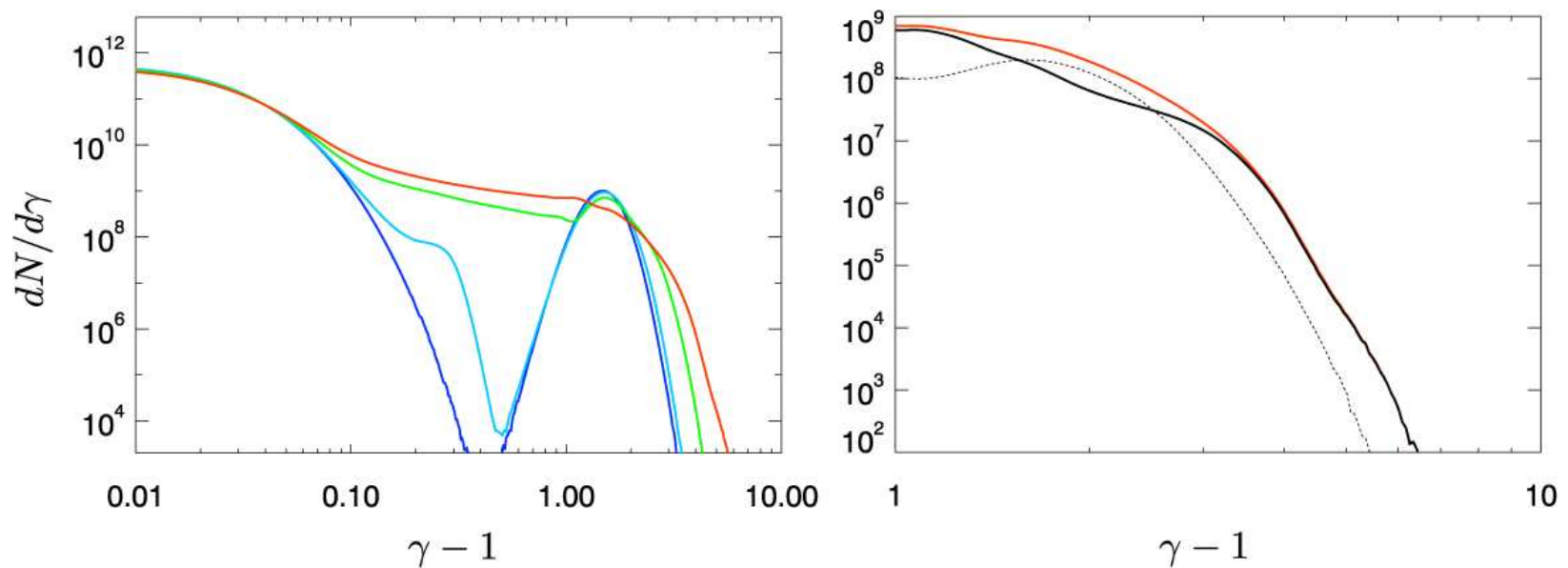

Figure 4: Left: Time evolution of the number spectra of the electrons at $t=0$ (blue), 20 (cyan), 250 (green), and $750 \omega_{\mathrm{pe}}^{-1}$ (red). Right: energy spectra of electrons at $t=750 \omega_{\mathrm{pe}}^{-1}$ in the range $1 \leq \gamma-1 \leq 10$. The spectrum of electrons in the whole simulation domain (red curve) is decomposed into those of electrons which currently exist inside/outside the initial shear surface (black solid-thick/dotted-thin curve). 
simulation domain is $(409.6 \times 409.6)$, where the unit of the length is the electron skin depth $c / \omega_{\text {pe }}$. This simulation domain is resolved by $(8192 \times 8192)$ grid cells, and the cell size is one-half of the Debye length of the electrons. The periodic boundary condition is imposed at each boundary of the computational domain.

Initially, the cylindrical jet is located at the center of the simulation domain and the diameter of the jet is set to be $100 \mathrm{c} / \omega_{\mathrm{pe}}$. Inside the jet region, the electrons and protons have bulk speed with $0.9 c$ in the $z$ direction, whereas the plasma has no bulk speed outside the jet. We assume that the uniform density plasma fills both inside and outside the velocity shear surface. The initial number of the computational particles, which are called superparticles, of both the electrons and the protons is 160 per grid cell. The temperatures of the electrons and protons are constant in space with its thermal velocity to be $0.1 c$ and $0.025 c$, respectively. These thermal velocities are defined in the co-moving frame of the bulk plasma flow. At first, the velocity of particles in the co-moving frame is determined to reproduce the Maxwell distribution by generating a set of random numbers based on a Mersenne-Twister method. Next, the four-velocity of particles is Lorentz boosted to the $z$ direction if the superparticles are inside the velocity shear surface, i.e., what is called shifted Maxwell distribution is realized inside the shear surface. We add no artificial initial perturbation to the plasma, i.e., the MI spontaneously grows by the electrons crossing the velocity shear surface due to their thermal motions. Initially, plasma has neither magnetic field nor electric current in the simulation domain. This assumption would be reasonable because the jet spine is detected in the region far from the central black hole $r \gtrsim 10^{3} r_{\mathrm{S}}$, in which at least a part of the magnetic fields is believed to be dissipated and their energy is converted to the kinetic energy of the jet. 
Since the injection mechanism of the plasmas into the jet is a long-standing, open question, we simply assumed the initially uniform density plasma composed of protons and electrons as described above. The protons and electrons might be injected into the jet base as a result of the MRs triggered at the interface of jet and turbulent accretion flow ${ }^{14}$ or the decay of the neutrons generated via the accelerated proton in the underlying accretion flows ${ }^{43}$. In our new formation mechanism of the jet spine, the high-energy electrons spontaneously concentrate into the jet center as a consequence of the MI-driven-MRs.

1. Frank, A. et al. Jets and Outflows from Star to Cloud: Observations Confront Theory. In Beuther, H., Klessen, R. S., Dullemond, C. P. \& Henning, T. (eds.) Protostars and Planets VI, 451 (2014). 1402.3553.

2. Blandford, R., Meier, D. \& Readhead, A. Relativistic Jets from Active Galactic Nuclei. ARA\&A 57, 467-509 (2019). 1812.06025.

3. Asada, K., Nakamura, M. \& Pu, H.-Y. Indication of the Black Hole Powered Jet in M87 by VSOP Observations. ApJ 833, 56 (2016).

4. Hada, K. The Structure and Propagation of the Misaligned Jet M87. Galaxies 5, 2 (2017).

5. Laing, R. A. \& Bridle, A. H. Relativistic models and the jet velocity field in the radio galaxy 3C 31. MNRAS 336, 328-352 (2002). astro-ph/ 0206215.

6. Ghisellini, G., Tavecchio, F. \& Chiaberge, M. Structured jets in TeV BL Lac objects and radiogalaxies. Implications for the observed properties. A\&A 432, 401-410 (2005). 
astro-ph/0406093.

7. Alves, E. P., Grismayer, T., Fonseca, R. A. \& Silva, L. O. Transverse electron-scale instability in relativistic shear flows. PhRvE 92, 021101 (2015). 1505.06016.

8. Hada, K. et al. An origin of the radio jet in M87 at the location of the central black hole. Nature 477, 185-187 (2011).

9. Walker, R. C., Hardee, P. E., Davies, F. B., Ly, C. \& Junor, W. The Structure and Dynamics of the Subparsec Jet in M87 Based on 50 VLBA Observations over 17 Years at $43 \mathrm{GHz}$. ApJ 855, 128 (2018). 1802.06166.

10. Event Horizon Telescope Collaboration et al. First M87 Event Horizon Telescope Results. I. The Shadow of the Supermassive Black Hole. ApJ 875, L1 (2019).

11. Koide, S., Shibata, K. \& Kudoh, T. Relativistic Jet Formation from Black Hole Magnetized Accretion Disks: Method, Tests, and Applications of a General RelativisticMagnetohydrodynamic Numerical Code. ApJ 522, 727-752 (1999).

12. McKinney, J. C. General relativistic magnetohydrodynamic simulations of the jet formation and large-scale propagation from black hole accretion systems. MNRAS 368, 1561-1582 (2006). astro-ph/0603045.

13. Mizuno, Y., Hardee, P. \& Nishikawa, K.-I. Three-dimensional Relativistic Magnetohydrodynamic Simulations of Magnetized Spine-Sheath Relativistic Jets. ApJ 662, 835-850 (2007). astro-ph/0703190. 
14. Tchekhovskoy, A., Narayan, R. \& McKinney, J. C. Efficient generation of jets from magnetically arrested accretion on a rapidly spinning black hole. MNRAS 418, L79-L83 (2011). 1108.0412.

15. McKinney, J. C., Tchekhovskoy, A. \& Bland ford, R. D. Alignment of Magnetized Accretion Disks and Relativistic Jets with Spinning Black Holes. Science 339, 49 (2013). 1211 . 3651.

16. Porth, O. \& Komissarov, S. S. Causality and stability of cosmic jets. MNRAS 452, 1089-1104 (2015). 1408.3318.

17. Gourgouliatos, K. N. \& Komissarov, S. S. Reconfinement and loss of stability in jets from active galactic nuclei. Nature Astronomy 2, 167-171 (2018). 1806.05683.

18. Nakamura, M. et al. Parabolic Jets from the Spinning Black Hole in M87. ApJ 868, 146 (2018). 1810.09963.

19. Davelaar, J. et al. Modeling non-thermal emission from the jet-launching region of M 87 with adaptive mesh refinement. A\&A 632, A2 (2019). 1906.10065.

20. Matsumoto, J. \& Masada, Y. Propagation, cocoon formation, and resultant destabilization of relativistic jets. MNRAS 490, 4271-4280 (2019). 1910.11578.

21. Nishikawa, K. I. et al. Particle Acceleration in Relativistic Jets Due to Weibel Instability. ApJ 595, 555-563 (2003). astro-ph/0305091.

22. Nishikawa, K. I. et al. Evolution of Global Relativistic Jets: Collimations and Expansion with kKHI and the Weibel Instability. ApJ 820, 94 (2016). 1511.03581. 
23. Alves, E. P., Zrake, J. \& Fiuza, F. Efficient Nonthermal Particle Acceleration by the Kink Instability in Relativistic Jets. PhRvL 121, 245101 (2018). 1810.05154.

24. Alves, E. P. et al. Large-scale Magnetic Field Generation via the Kinetic Kelvin-Helmholtz Instability in Unmagnetized Scenarios. ApJ 746, L14 (2012). 1107.6037.

25. Grismayer, T., Alves, E. P., Fonseca, R. A. \& Silva, L. O. dc-Magnetic-Field Generation in Unmagnetized Shear Flows. PhRvL 111, 015005 (2013). 1205.2293.

26. Liang, E., Boettcher, M. \& Smith, I. Magnetic Field Generation and Particle Energization at Relativistic Shear Boundaries in Collisionless Electron-Positron Plasmas. ApJL 766, L19 (2013). 1111.3326.

27. Alves, E. P., Grismayer, T., Fonseca, R. A. \& Silva, L. O. Electron-scale shear instabilities: magnetic field generation and particle acceleration in astrophysical jets. New Journal of Physics 16, 035007 (2014). 1404 . 0555.

28. Moritaka, T., Kuramitsu, Y., Liu, Y.-L. \& Chen, S.-H. Spontaneous focusing of plasma flow in a weak perpendicular magnetic field. Physics of Plasmas 23, 032110 (2016).

29. Parker, E. N. Cosmical magnetic fields. Their origin and their activity (1979).

30. Horiuchi, R. \& Sato, T. Three-dimensional particle simulation of plasma instabilities and collisionless reconnection in a current sheet. Physics of Plasmas 6, 4565-4574 (1999).

31. Biskamp, D. Magnetic Reconnection in Plasmas, vol. 3 (2000). 
32. Zenitani, S. \& Hoshino, M. The Generation of Nonthermal Particles in the Relativistic Magnetic Reconnection of Pair Plasmas. ApJL 562, L63-L66 (2001). 1402 . 7139.

33. Drake, J. F., Swisdak, M., Che, H. \& Shay, M. A. Electron acceleration from contracting magnetic islands during reconnection. Nature 443, 553-556 (2006).

34. Yamada, M., Kulsrud, R. \& Ji, H. Magnetic reconnection. Reviews of Modern Physics 82, 603-664 (2010).

35. Sironi, L. \& Spitkovsky, A. Relativistic Reconnection: An Efficient Source of Non-thermal Particles. ApJL 783, L21 (2014). 1401.5471.

36. Ishizawa, A. \& Horiuchi, R. Suppression of Hall-Term Effects by Gyroviscous Cancellation in Steady Collisionless Magnetic Reconnection. PhRvL 95, 045003 (2005).

37. Event Horizon Telescope Collaboration et al. First M87 Event Horizon Telescope Results. V. Physical Origin of the Asymmetric Ring. ApJ 875, L5 (2019).

38. Asada, K. \& Nakamura, M. The Structure of the M87 Jet: A Transition from Parabolic to Conical Streamlines. ApJL 745, L28 (2012). 1110.1793.

39. Nakamura, M. \& Asada, K. The Parabolic Jet Structure in M87 as a Magnetohydrodynamic Nozzle. ApJ 775, 118 (2013). 1308.1436.

40. Birdsall, C. K. \& Langdon, A. B. Plasma Physics via Computer Simulation (1991).

41. Hockney, R. W. \& Eastwood, J. W. Computer simulation using particles (1988). 
42. Esirkepov, T. Z. Exact charge conservation scheme for Particle-in-Cell simulation with an arbitrary form-factor. Computer Physics Communications 135, 144-153 (2001).

43. Toma, K. \& Takahara, F. Baryon Loading of Active Galactic Nucleus Jets Mediated by Neutrons. ApJ 754, 148 (2012). 1205.6868.

44. Speiser, T. W. Particle Trajectories in Model Current Sheets, 1, Analytical Solutions. J. Geophys. Res. 70, 4219-4226 (1965).

45. Horiuchi, R. \& Ohtani, H. Formation of Non-Maxwellian Distribution and Its Role in Collisionless Driven Reconnection. Communications in Computational Physics 4, 496-505 (2008).

Acknowledgements We thank M. Kino, E. Kokubo, K. Asada, K. Hada, and M. Toida for useful discussion. This research was funded by the NINS program of Promoting Research by Networking among Institutions (Grant Number 01421701). Numerical calculation was performed on the Plasma Simulator (FUJITSU FX100) of NIFS with the support and under the auspices of the NIFS Collaboration Research program (NIFS17KNSS092). A part of the simulations are performed on the XC30 at the Center for Computational Astrophysics in NAOJ.

Author contributions T.K. performed PIC simulations, analyzed most of simulation data, and wrote the original draft. T.K., S.I. and K.T. formulated the idea of this paper. T.M. provided the PIC simulation code PASTEL, helped to perform PIC simulations and to analyze the result, and analyzed a part of simulation data. R.H. provided many ideas for the analysis of the simulation results, especially on the magnetic reconnection. S.I., R.H. and K.T. initiated and supervised the project funded by the NINS program. All authors discussed the results and contributed to reviewing and editing the manuscript. 
Competing interests The authors declare that they have no competing financial interests.

Correspondence Correspondence and requests for materials should be addressed to Tomohisa Kawashima (email: kawshm@icrr.u-tokyo.ac.jp).

\section{Supplementary information}

Mushroom instability

The MI is the relativistic, electron-scale shear instability. Due to the generation of the magnetic field and the enhanced interchange of electrons due to the resultant Lorentz force, the morphology of the deformed electron shear surface shows mushroom like structure, which is demonstrated in Figure 5.

At the early stage of our global simulation $\left(\sim 1 / \omega_{\text {pe }}\right)$, the MI starts to grow near the velocity-shear surface. This timescale is consistent with the growth timescale of MI assuming cold plasma ${ }^{7} t_{\mathrm{MI}} \sim\left[(\bar{v} / c) \sqrt{\bar{\gamma}} \omega_{\mathrm{pe}}\right]^{-1}$, where $\bar{v}$ and $\bar{\gamma}$ are the velocity and the Lorentz factor of the shear flow measured in the moving frame in which we observe the shear speed as $+\bar{v}$ and $-\bar{v}$. Our jet model with $v_{\text {bulk }}=0.9 c$ corresponds to $\bar{v} \sim 0.6 c$, where these are related by $v_{\text {bulk }}=2 \bar{v} /\left(1+\bar{v}^{2} / c^{2}\right)$ and $\bar{\gamma} \simeq 1.2$, so that $t_{\mathrm{MI}} \sim 1 / \omega_{\text {pe. }}$ One can find that the MI linearly grows in this timescale in the left panel of Figure 6. Because the thermal velocity of electrons is higher than that of the protons, the electrons easily cross the velocity shear surface of the jet rather than protons. This electron motion leads to the generation of electric current in the vicinity of the velocity shear surface. Subsequently, the magnetic fields surrounding the jet spine are generated near the shear surface. Then, the electrons experience the Lorentz force, which enhances the electron motion to cross the shear surface, and the perturbation grows into a number of electron channel-flows. The mushroom like morphology appears in the head of the channel flows, and this is the basic behavior of electrons in the linear stage of MI. 
In the non-linear stage of the MI, the magnetic reconnection (MR) is recurrently induced in the shear-flow region (that is, MI-driven-MR). The channel flow accompanies the strong electric current and the antiparallel configuration of the magnetic field elongated in the radial direction. Then, the MR is triggered near the shear surface and the heated and/or accelerated electrons are ejected towards the regions inside and outside the shear surface, as shown in the main text. At the early non-linear stage, the electromagnetic energy intermittently varies. This is due to the episodic MI-driven-MR; the MRs convert the electromagnetic energy amplified by the MI to the electron energy, while the MI recovers the magnetic energy lost by the MRs and forms new electric current sheets followed by subsequent MRs. In the later non-linear stage of MI, the electromagnetic energy is amplified up to $10^{-4}$ of the initial particle energy in the whole simulation domain. Throughout the simulation, the magnetic energy dominates the electric energy since the simulated plasma is mildly relativistic. These are shown in the right panel of Figure 6.

\section{Hole structure in Magnetic reconnection}

Here, we confirm that the MRs are physically triggered in our simulation. Figure 7 displays a phase-space $\left(y-v_{y}\right)$ plot of electrons, and one can clearly find the appearance of the hole structure at around $y \simeq 3$ and

$v_{y} \simeq 0$. This hole structure in the phase space is a consequence of the stochastic motion of electrons in the vicinity of the magnetic neutral sheet, which is called "meandering motion" 44 . The hole structure in the phase space is a strong evidence of the MRs ${ }^{45}$.

\section{supplementary movie}

The movie 1 shows the time evolution of the synchrotron emissivity mimicked by displaying the number density of the electrons multiplied by the magnetic field strength as is shown in Figure 1. As time increases, the jet center becomes bright because of the concentration of electrons towards the jet center (see movie 2) 

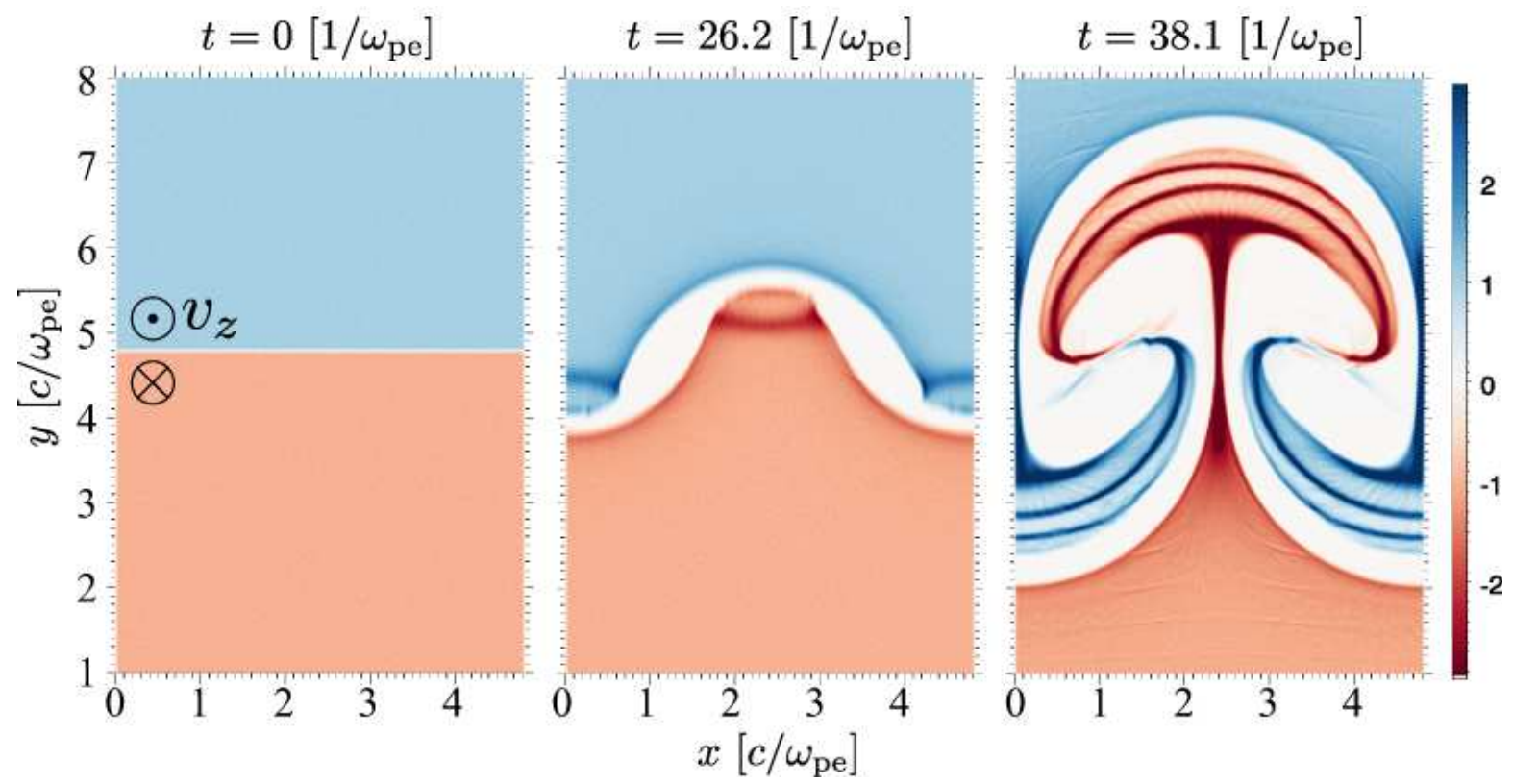

Figure 5: (supplementary information) Time evolution of MI of electron-proton plasma with real mass ratio in the cold plasma limit. The colour displays the dye concentration field of electrons. In the region shown in this figure, the blue/red colour is initially attached to the electrons in the upper/lower side of the velocity shear surface at $y=4.8 \mathrm{c} / \omega_{\mathrm{pe}}$. Then, the dynamics of electrons are solved via the PIC simulations with their colour information being kept. White coloured region means that the red- and blue-coloured electrons are mixed there. The initial bulk velocity of protons and electrons is $v_{z}=+0.5 \mathrm{c}$ and $-0.5 \mathrm{c}$ above and below the velocity shear surface, respectively. The simulation domain is $L_{x} \times L_{y}=4.8\left[\mathrm{c} / \omega_{\mathrm{pe}}\right] \times 19.2\left[\mathrm{c} / \omega_{\mathrm{pe}}\right]$, which is divided by $192 \times 768$ grid cells, i.e., the initial electron skin depth is resolved by 40 grid cells. The density and thermal velocity of protons and electrons are initially uniform, except the $y$-component of velocity is perturbed in such a way that $v_{y}=-\left(10^{-4} c\right) \cos \left(2 \pi x / L_{x}\right)$. The periodic boundary condition is imposed in $x$ and $y$ directions. We note that roughly the lower half of the simulation domain is shown in this figure. 

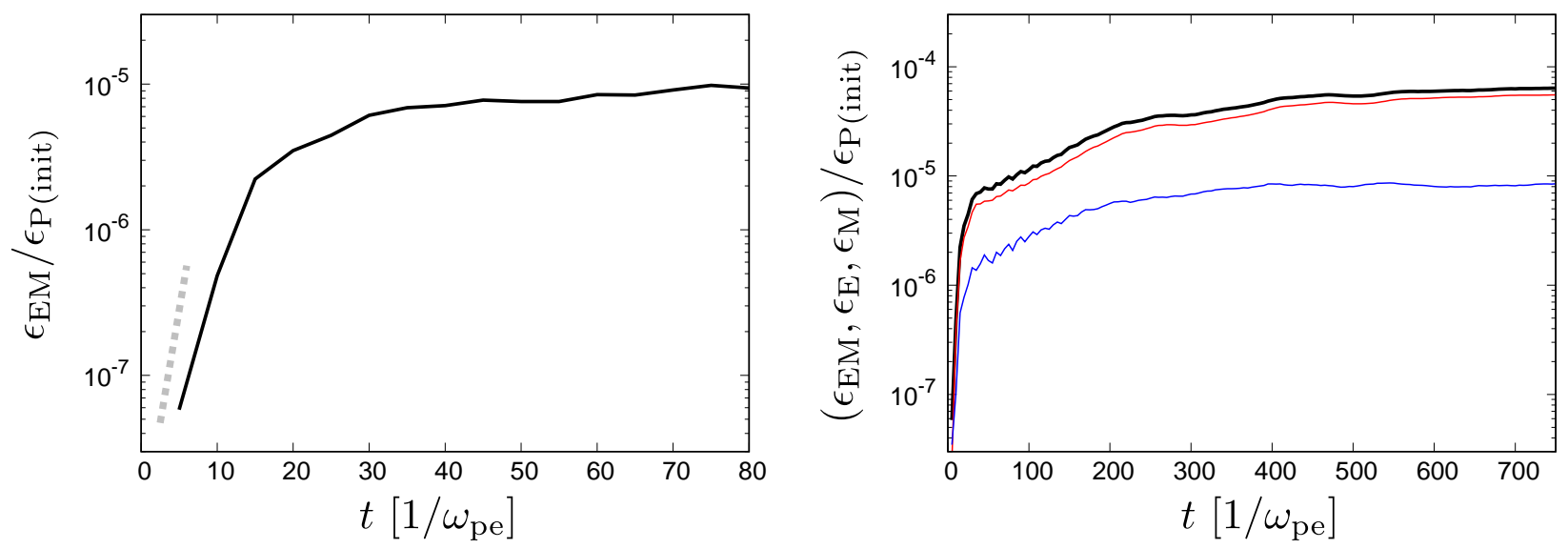

Figure 6: (supplementary information) Time evolution of energy of electromagnetic field $\epsilon_{\mathrm{EM}}$, electric field $\epsilon_{\mathrm{E}}$, magnetic field $\epsilon_{\mathrm{M}}$ normalized by initial kinetic energy of particles (i.e., protons and electrons) $\epsilon_{\mathrm{P}(\text { init) }}$, which are measured in the laboratory frame. The left panel shows the early stage of simulation. The black curve represents $\epsilon_{\mathrm{EM}}$. As a reference, the slope of maximal growth rate of MI $(\propto \bar{v} / c \sqrt{\bar{\gamma}})$ for cold plasmas is shown by the gray dashed line. The MI grows linearly

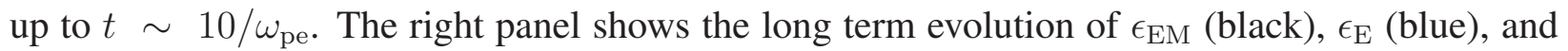
$\epsilon_{\mathrm{M}}(\mathrm{red})$. 


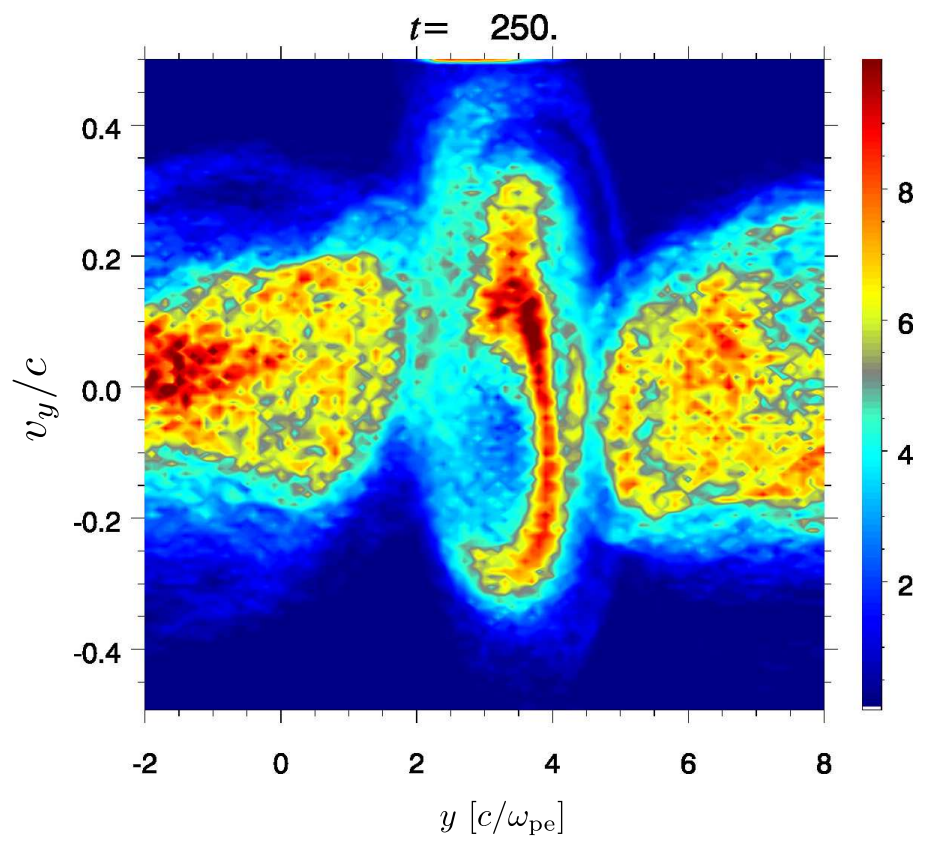

Figure 7: (supplementary information) The electron distribution in $y-v_{y}$ phase space at $t=$ $2.5 \times 10^{2} \omega_{\mathrm{pe}}^{-1}$, in which electrons are sampled along the line $x=46 c / \omega_{\mathrm{pe}}$. The hole structure at $\left(y, v_{y} / c\right) \simeq(3,0)$ evidently presents the appearance of the MR at this point. In this figure, the velocity in $y$-direction is evaluated in the co-moving frame of the bulk motion in $z$-direction. 
and the amplification of the magnetic field. This movie demonstrates the formation of the jet spine, which is observed as a bright beam in the radio image of M87.

The movie 2 represents the evolution of the electron number density in the whole simulation domain, of which the snapshots are shown in figures 1 and 2. As the time increases, the electrons concentrate to the jet center.

The movie 3 displays the time evolution of the electron density, the electron velocity in the transverse direction to the jet, the $z$-component of the electric current density, and the product of the $z$-component of the electric current and electric field, in the region near the initial velocity shear surface. One can find that the recurrent magnetic reconnection, which is triggered by the Mushroom instability, occurs and energize the electrons. 


\section{Figures}

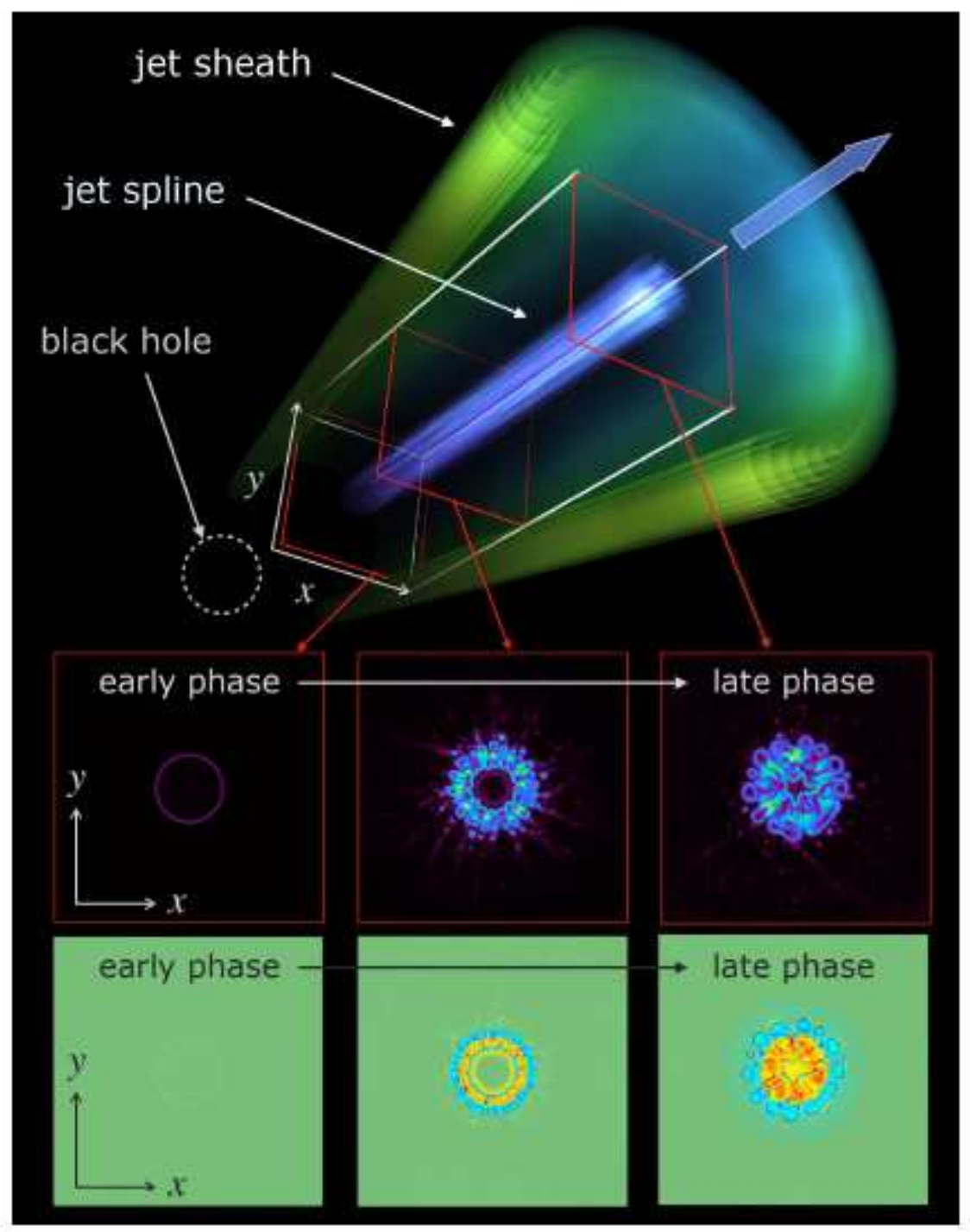

\section{Figure 1}

Synthetic picture of our relativistic jet model. The top panel is composed of the volume rendered image of our PIC simulation (jet spine, which is colored in blue-green-white) and schematic pictures (jet sheath colored in green). This image is synthesized by stratifying the time snapshots of our 2D PIC simulation in the direction of the jet propagation. The color represents the electron density multiplied by the strength of the magnetic field, which roughly agrees with the synchrotron emissivity, which is the most important radiative process in the $\mathrm{M} 87$ jet. The middle and bottom panels show the spatial distribution of the electron-density multiplied by the strength of the magnetic field the above pseudo synchrotron emissivity and the electron-density itself only, respectively, at each epoch. (animation: supplementary material) 

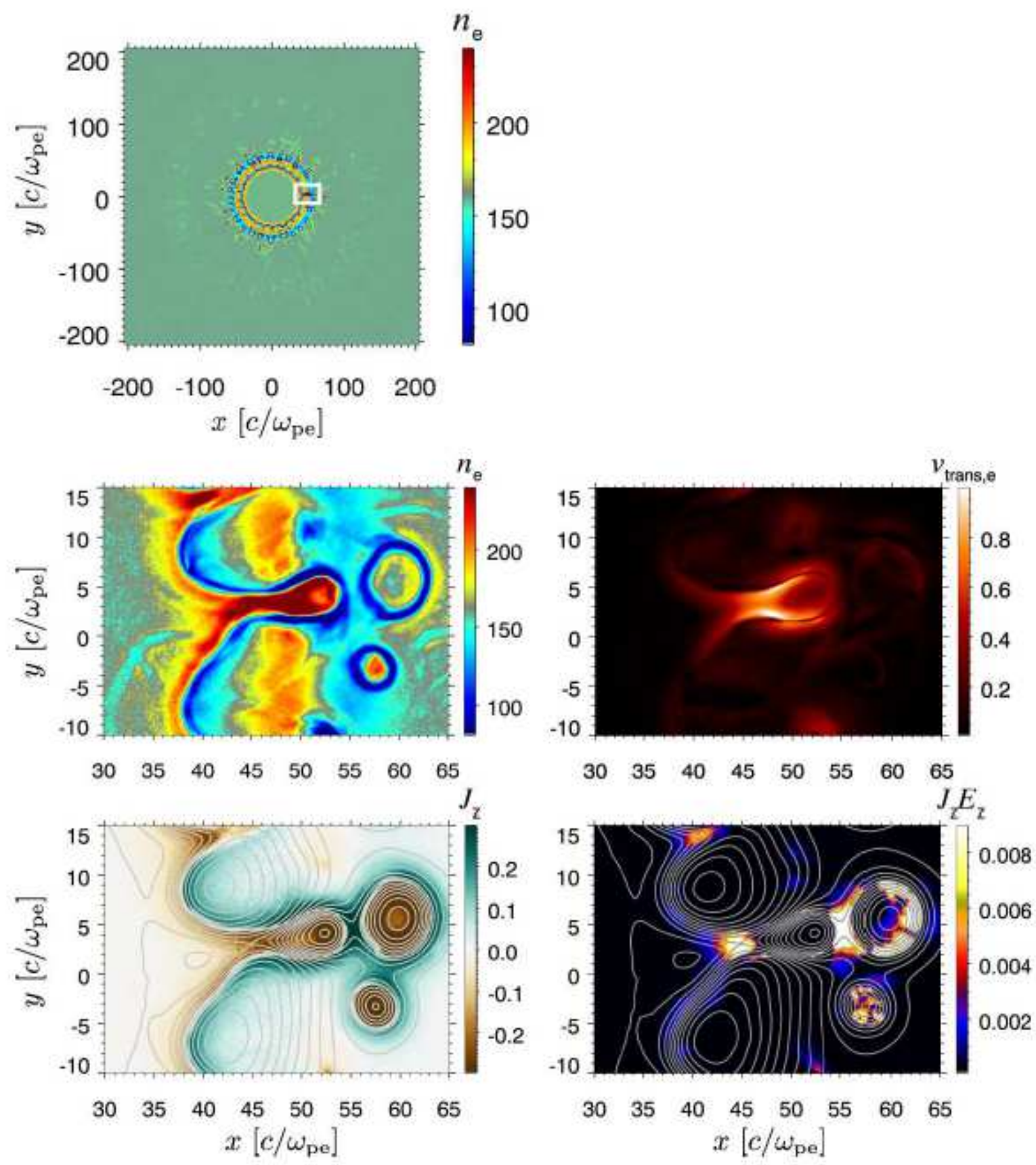

\section{Figure 2}

Electron density map in the whole simulation domain (top) at $t=2.5 \times 102 \omega-1$ pe. Magnified view of the electron density (middle left), the absolute value of the four-vector of the electron velocity in the transverse direction of the jet (middle right), $z$ component of electric current density (bottom left), and the product of z component of electric current density and electric field (bottom right) in the region enclosed in the top panel. The electric current and the electric field are evaluated in the co-moving frame of the electrons at each point. The gray lines in the bottom panels display the magnetic field lines in the plane. (animation: supplementary material) 


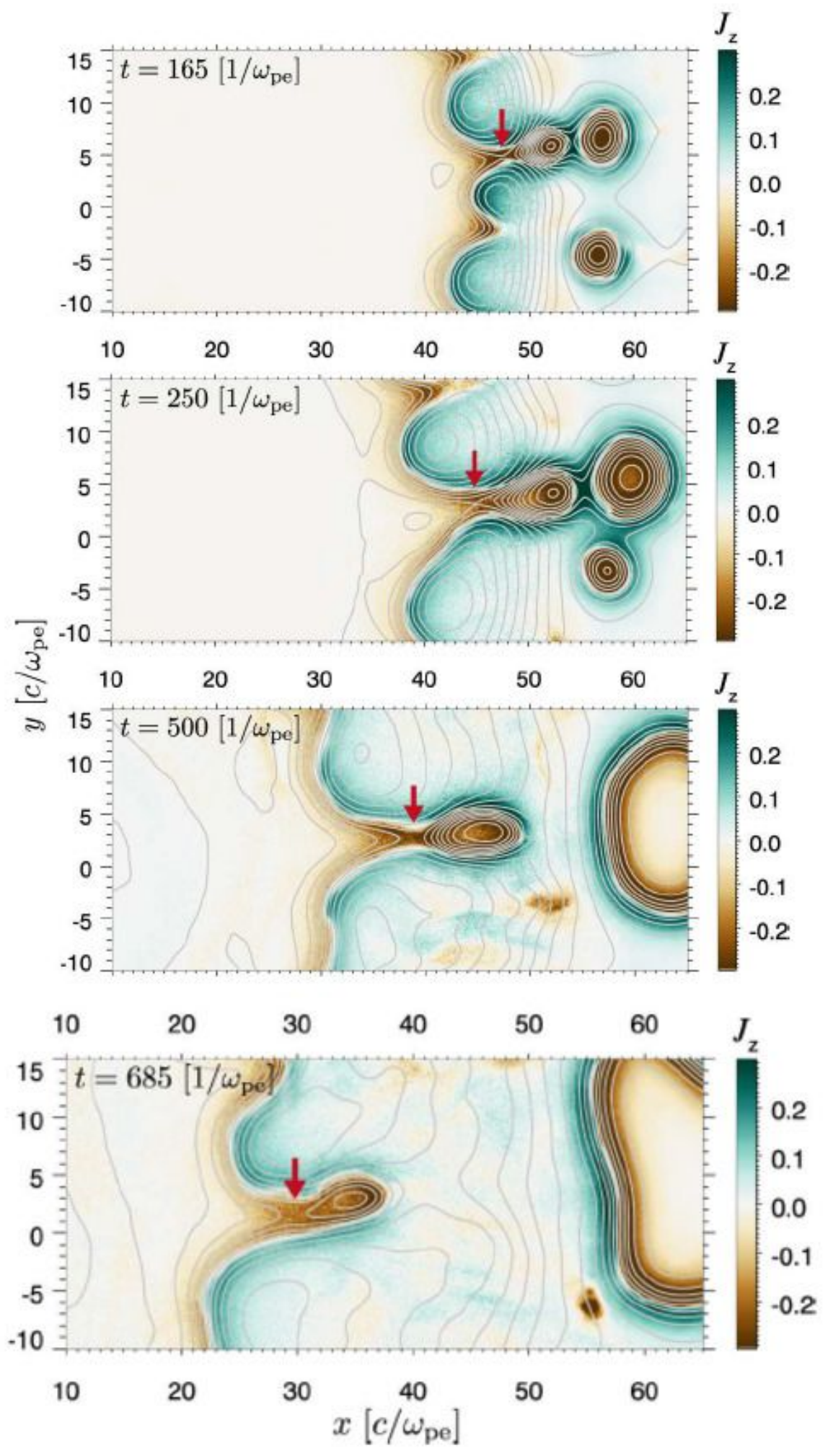

Figure 3

Time evolution of the MI-driven-MR near shear surface. The electric current sheet and the reconnection points (red arrows) shift towards the jet center with time. The electric current is evaluated in the comoving frame of electrons at each point. 

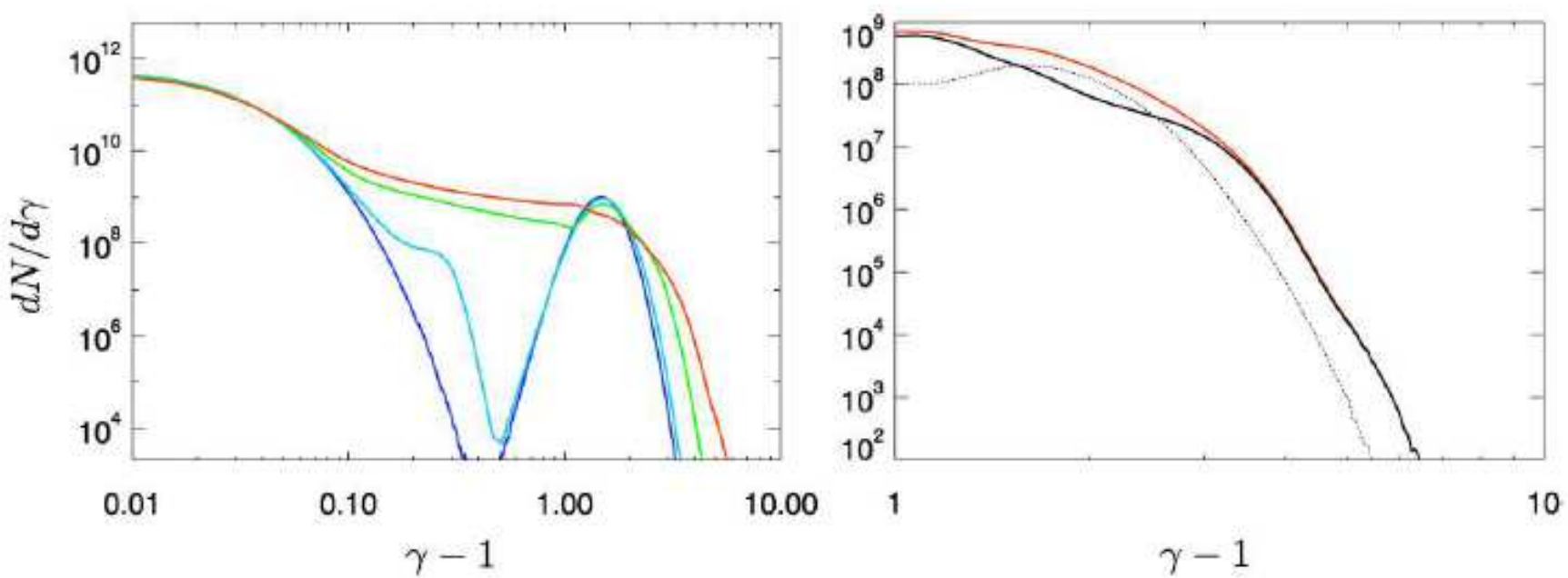

\section{Figure 4}

Left: Time evolution of the number spectra of the electrons at $t=0$ (blue), 20 (cyan), 250 (green), and 750 $\omega-1$ pe (red). Right: energy spectra of electrons at $t=750 \omega-1$ pe in the range $1 \leq \gamma-1 \leq 10$. The spectrum of electrons in the whole simulation domain (red curve) is decomposed into those of electrons which currently exist inside/outside the initial shear surface (black solid-thick/dotted-thin curve).

\section{Supplementary Files}

This is a list of supplementary files associated with this preprint. Click to download.

- movie2.mov

- movie3.mov

- movie1.mp4

- Fig5supp.png

- Fig6supp.png

- Fig7supp.png 